\title{
Sophie Podolski, maudite petite Belge
}

\author{
Denis St-Amand \\ FNRS - Université de Liège
}

En réalité, lire est toujours plus important qu'écrire. Roberto Bolaño

S'il est une auteure que les lecteurs de Roberto Bolaño ont appris à connaître, c'est bien Sophie Podolski, curieuse étoile filante dont les passages fulgurants au cœur des romans et nouvelles du Chilien font sens à force de répétition. Digne de figurer dans les bibliothèques idéales de différents protagonistes mis en scène par Bolaño (aventuriers sans-le-sou, clochards célestes, apprentis écrivains et autres spectres 
exhalant un fort parfum d'autofictif), Podolski n'est toutefois convoquée qu'à titre d'absente. De l'aventure, d'abord, puisqu'elle n'est en somme qu'un élément métonymique au sein de listes de livres ou de souvenirs de lectures, mais aussi, et surtout, de la vie, puisque l'auteur manque rarement de rappeler sa qualité de jeune suicidée. Cette omniprésente absente, plus qu'un détail encyclopédique, se révèle un véritable composant fictionnel, convoqué pour irradier symboliquement sur le récit et nourrir l'imaginaire de ce dernier. Dans Les Détectives sauvages, on trouve de cette façon une mention du recueil de Sophie Podolski, Le Pays où tout est permis, coincé sous le bras d'un personnage entre Le Parfait criminel de Jouffroy et Cent mille milliards de poèmes de Queneau (Bolaño, 2010, p. 39)ํ․ Dans la nouvelle «Vagabonds en France et en Belgique », insérée dans le recueil Des Putains meurtrières, le héros-vagabond, désigné par la seule lettre $\mathrm{B}$, est un lecteur assidu de la revue Luna Park, où le nom de l'écrivaine belge côtoie ceux de Christian Dotremont, de Pierre Guyotat et de Roland Barthes. Consacrant quelques lignes à chacun de ces auteurs, Bolaño précise que «Sophie Podolski a été une poétesse que [B] et son ami L ont appréciée (et même pourraiton dire ont aimée) au Mexique, quand $\mathrm{B}$ et $\mathrm{L}$ vivaient à Mexico, et avaient à peine plus de vingt ans » (2003, p. 99). L'un des chapitres logorrhéiques d'Anvers, encore, lui rend hommage qui devient, dans la traduction de Robert Amutio, une véritable bulle de pastiche que la reprise finale d'un motif inaugural clôt sur elle-même, en une quasi-épanalepse :

\footnotetext{
1 Dans un billet datant de 2010, l'auteur du blog littéraire anglophone « Avant que j'oublie » avait déjà passé en revue différentes références à Podolski dans l'œuvre de Bolaño. Sur différents documents relatifs à l'auteure, voir Gauvin, 2009.
} 
L'enfer qui viendra... Sophie Podolski s'est suicidée il y a plusieurs années... Elle aurait aujourd'hui vingt-sept ans, comme moi... Des patrons égyptiens au plafond uni, les employés s'approchent lentement, des champs poussiéreux, nous sommes à la fin avril et on les paie avec de l'héroïne... J'ai allumé la radio, une voix impersonnelle procède à la recension par bille des personnes arrêtées ce jour... « Jusqu'à zéro heure, aucune nouvelle »... Une jeune fille qui écrivait des dragons, complètement pourrie dans une niche de Bruxelles... «Mitraillettes, pistolets, grenades confisqués »... Je suis seul, toute la merde littéraire est restée derrière, revues de poésie, éditions limitées, toute cette plaisanterie grise est restée derrière... Le type a ouvert la porte au premier coup de pied et t'a mis le pistolet sous le menton... Bâtiments abandonnés de Barcelone, presque une invitation à se suicider en paix... Le soleil derrière le voile de poussière dans le crépuscule près du Nil... Le patron paie avec de l'héroïne et les paysans sniffent dans les sillons, écroulés sur les couvertures, sous des palmiers écrits que quelqu'un corrige et fait disparaitre... Une jeune fille belge qui écrivait comme une étoile... « Elle aurait aujourd'hui vingt-sept ans, comme moi »... (Bolaño, 2004, p. 29-30)

Énoncé comme une parole vive, ce petit portrait dithyrambique de l'écrivaine en estrella - ou en hopeful monster, peut-être ${ }^{2}-$

\footnotetext{
2 L'expression est reprise par Fresán pour évoquer l'une de ses conversations avec Bolaño : "Je me souviens aussi qu'à un moment, cet après-midi, a surgi dans la conversation le thème des hopeful monsters; ceux que les biologistes et généticiens nomment les monstres espérants ou optimistes. [...] Des créatures qui ne sont rien moins qu'une mutation. Une variante hors programme dans le répertoire de l'espèce. Une exception qui peut se renforcer jusqu'à devenir une bête dominante ou victorieuse - d'où son espoir, son optimisme - ou, après un temps, succomber et disparaître sans laisser de trace comme les dragons, les fées, ou les licornes. En de rares occasions, les monstres espérants se débrouillent pour perdurer et se mêler aux gens "normaux", parfois disposés à s'adapter aux modes et aux nécessités du monde. Mais cela n'arrive généralement pas. Les monstres espérants ont tendance à lutter jusqu'au bout pour essayer de devenir la nouvelle règle immuable et obliger le monde à s'adapter à leurs nouveaux traits et habitudes. Petit à petit, ils périssent, victimes du lyrisme de leur inimitable ambition. »
} 
fait autant écho, on va le constater, à la fascination de la jeune femme pour les drogues qu'à une façon de mise en scène posturale de cette dernière en maudite virulente ${ }^{3}$. Mais qui, au juste, est cette Sophie Podolski, qu'on serait presque tenté de croire sortie directement de l'imagination de Bolaño ? C'est que, si elle obsède le regretté romancier, l'éternelle absence qui la caractérise dans la fiction semble également valoir pour l'histoire littéraire: les plus complets essais et anthologies consacrés à la littérature belge francophone ne la mentionnent jamais, et on ne trouve guère d'articles scientifiques à son sujet.

\section{"Parce que c'est ici une planète de cons"}

La poétesse a pourtant bel et bien vécu, et n'est pas tout à fait insaisissable : en la présentant dans Le Dictionnaire littéraire des femmes de langue française, l'écrivaine Françoise Collin précise qu'elle est née le 8 octobre 1953 et s'est suicidée le 23décembre 1974, à Bruxelles (1996, p. 473-476). Au cours de cette trajectoire-éclair, Sophie Podolski a en outre "sub[i] de brefs internements psychiatriques à Bruxelles et à Paris » (Collin, 1996, p. 474). Elle est l'auteure d'une seule œuvre publiée de son vivant, Le Pays où tout est permis. Ce texte, dont le manuscrit est conservé aux Archives et Musée de la littérature de la Bibliothèque royale de Bruxelles, a fait l'objet d'une première publication aux éditions Montfaucon

(2012, p.62.) Il n'est pas impossible, comme on s'en rendra compte, que Podolski ait été citée dans cette parade de monstres espérants.

3 Sur la notion de posture comme "manière d'occuper une position dans le champ » appréhendable par des discours et des comportements, voir Meizoz, 2007 et 2011 ; COnTEXTES, 2011. 
(Bruxelles) en 1972. Il est ensuite réédité deux ans plus tard chez Belfond et accompagné pour l'occasion d'une préface de Philippe Sollers, qui en avait auparavant proposé des extraits dans les 53e et 54e livraisons de Tel Quel. Sollers, dans la revue, introduisait l'auteure de la façon suivante : "Sophie Podolski est née en 1953. Cette date permet de mesurer la rapidité avec laquelle un certain bouleversement est en cours. Et en guise d'avertissement pour ceux qui croient qu'écrire ne vient pas d'abord d'une certaine façon de vivre. » (1973, n. p.). L'œuvre de la jeune femme, sorte de journal extime chaotique, est fidèle à la trajectoire de celle-ci : une anomie délibérée y est affirmée avec violence au cours d'un monologue halluciné et volontiers digressif. Chercher à en rendre compte dans sa totalité, en plus de nécessiter un large espace, serait coûteux sur le plan paradigmatique, en ce sens que les sujets posés par ce texte déstructuré sont au fond presque aussi nombreux que les pages qui le composent. Dès son ouverture, le poème de Podolski laisse toutefois transparaître quelques mots d'ordre thématiques et stylistiques :

Les cheveux du soleil sont nos mains aussi.

L'écriture pompiérise tout signe alarme continue.

Lettre à tous les mondes. Vous êtes tous des cons - ou bien vous êtes pas défoncés ou vous flipez comme des cons - parce que c'est ici une planète de cons qu'on comprendra jamais et on y comprend rien à rien. Est-elle femme ou démon. Est-ce démon ou femme - le suicide (développement) philosophiquement jamais étudié - pensez à dialoguer en vue de synthèses (perspective vraie ou fausse) ce que la parole avec la pensée n'a rien à voir avec notre organisme d'aucun autre fonctionnement de notre connaissance. La parole est une hystérie qui relève de la frustration qui par ailleurs la compense. Vous êtes si loin. Soyez sage - planant — à bientôt — l'herbe est dans le tiroir - il faut que vous sachiez rudement 
bien ce qu'il va advenir de vous pour que vous soyez si peu sûr de vous? $\left(1973\right.$, p. 15) ${ }^{4}$

Réfractaire à la syntaxe classique, privilégiant la note à l'exposé et toute déployée en parataxe, l'écriture peut sembler ici osciller entre l'automatisme prôné par les surréalistes et le cahier de brouillon. La drogue («Soyez sage — planant l'herbe est dans le tiroir »), la mort (le suicide, concept et réalité qui obnubileront Podolski jusqu'à la consommation, est d'emblée présent) et l'invective globalisée («vous êtes tous des cons ", «parce que c'est ici une planète de cons », insultes dont l'extrême universalité rend quasi dérisoire la portée irrévérencieuse) constituent trois piliers d'un délire agressif qui confine à la folie. Relativement classiques du côté de certaine bohème post-sixties goûteuse de psychotropes et révoltée contre tout ce qui se peut, ces trois grandes thématiques, qui se nourrissent les unes les autres, réapparaissent tout au long du texte. Ce dernier, par ailleurs, hésite à choisir sa cible :

Défoncez-vous les uns les autres - il n'est pas nécessaire d'avoir un jus de citron pur avec de la glace et du sucre et un joint académique avec un long filtre arôme mentholé défoncez-vous la gueule les uns les autres il n'est pas besoin de cachette ni de flipage collectif - deux lèvres comme deux filets d'une matière plastique quelconque - de même pour le dito le chat hérissé comme une angoisse encombrante - tu ne fumes pas le joint avec moi — je suis là — je suis là pas ici. (Podolski, 1973, p. 17)

La séquence injonctive qui ouvre l'extrait susmentionné, professée comme une quasi-parodie de discours biblique, fonctionne à la fois comme un commandement hallucinatoire destiné à l'ensemble de l'espèce humaine (qui devient plus loin

\footnotetext{
4 Nous respectons les graphie et orthographe (en partie corrigées) de cette édition, au détriment de celles du manuscrit original.
} 
un « défoncez-vous la gueule les uns les autres » plus belliqueux que narcotique) et comme une parole autoritaire, dont l'énonciateur est difficilement saisissable. Plus loin dans l'extrait, toutefois, un je échange un dialogue intime avec un tu muet - débarqué, lui aussi, d'on ne sait trop où - au cours d'une partie de cache-cache rendue floue par les effluves et les effets d'un «joint» omniprésent. Ailleurs, ce sont des lieux communs à valeur axiomatique qui contaminent l'énoncé, comme lorsque l'énonciateur prend, par exemple, l'armée à partie, dont les membres sont réduits à des imbéciles frustrés :

tous les soldats ne sont ni courageux - ni fiers - ni guerriers téméraires - quelques-uns sont téméraires - tous sont crétins. Ils auraient voulu connaître la petite guerre ils sont déçus. (Podolski, 1973, p. 19)

En d'autres lieux encore se relèvent quelques bribes d'envolées relativement cohérentes. En témoigne l'extrait suivant, qui, bien que porté par une forme de glossolalie, est irrigué par une isotopie carcérale où se croisent l'hôpital et la prison, en une description de la dépendance à la drogue dans laquelle se lit tout l'état de déréliction de l'énonciateur :

vous m'avez pris mon joint - now I go smoke a littl' pipe sunshine strawberry - une pipe de reine une pipe de DIEU à mort les canaris - les bambinos - les seringues en panne là — dancing - c'est le temps pour danser. Je suis guérie vous m'avez envoûtée et êtes passible d'amendements - vous n'aviez qu'à dire que vous aviez dépassé la ligne jaune - les cavaliers dans les falaises disparaîtront - ce sont des paroles verbales - non c'est une diarrhée vervale $[s i c]$ - je ne veux plus connaître de mots - je n'arrive pas à ne plus en connaître - nous ne sommes pas des animaux - nous sommes des hommes tu prends tes tournants trop larges - le pied - le pied - quand même elle a une voix - la table d'op roulant dans le couloir verdâtre carrelé blanc - cette odeur d'éther le pied - le pied - le bruit de l'ambulance me reste dans les 
oreilles - qui a commencé la bagarre - appeler le geôlier défaites ces nœuds - enlevez ces baxters - faites un joint allez faites un joint - on crève ici alors va chier. AHHH elle est vicieuse - elle parle de noisettes - travaillez pour nous avec quels pieds quelles mains - (tu vois bien que je veux vivre encore) - pas crever maintenant - ACID - SPEED HEROINE - quelles sont les drogues libres seulement morphine - même pas d'opium - ce n'est pas vrai - cela n'a plus d'importance [...]. (p. 22-23)

Rompant fréquemment la ligne autobiographique liée à la pratique diariste ${ }^{5}$, le manuscrit de Podolski est toutefois lisible comme un quasi-journal toxicomane et adolescent, dans lequel le je se met en scène en position de faiblesse mêlée de lucidité : en somme, ce qui y est affirmé, c'est que cette vie médiocre n'est pas simple et que l'auteure a compris que les paradis artificiels permettaient au moins d'échapper partiellement à cette "planète de cons ». Les dessins qui émaillent le petit cahier de Podolski, reproduits dans l'édition livresque, prolongent l'argument de ces prises de position désenchantées.
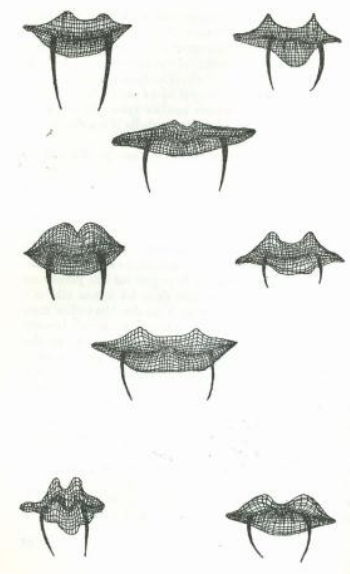

5 Voir à ce sujet Lejeune, 2005, et Simonet-Tenant, 2001. 


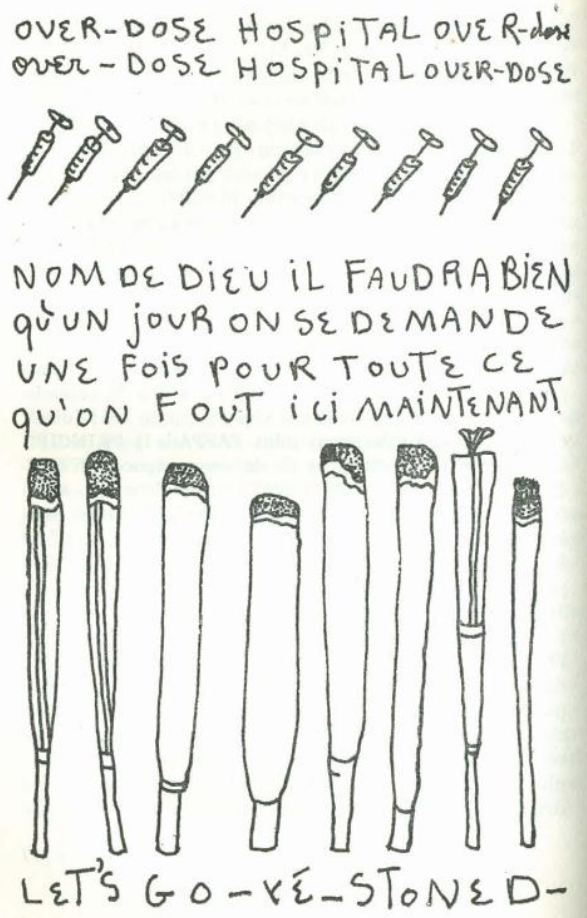

En accordant à ces échanges entre texte et image une place de choix, l'édition s'inscrit dans le sillage des ouvrages collaboratifs de la période surréaliste mêlant poésie et illustration, qu'elle cherche sans doute à rappeler (qu'on songe, par exemple, à La Clef des champs de Breton et de Miró). Figurent ici, entre autres, une série de huit bouches de vampires pulpeuses (Podolski, 1973, p. 76) et un échantillon de seringues et de joints exhibés comme autant de palliatifs violents d'une existence absurde («nom de dieu il faudra bien qu'un jour on se demande une fois pour toute ce qu'on fout ici maintenant», Podolski, 1973, p.118). Ailleurs, c'est l'écriture 
www.revue-analyses.org, vol. 8, no 1, hiver 2013

même de Podolski qui est reproduite, tantôt dépouillée et minimaliste tant sur le plan argumentatif que typographique (ainsi, à la page 150, du vaste programme « Ma foi... Milles scorpions!!! Je casserais bien la gueule à tous les gens du monde moi!», suivi de la signature d'une pseudonymique «Odile Croc » et de la numérotation du petit cahier), tantôt mêlant différents niveaux d'énonciation et différents jeux de caractères (comme dans l'exemple, difficilement transcriptible, de la page 152).

Noct Foisss Milles Seropions!!!!
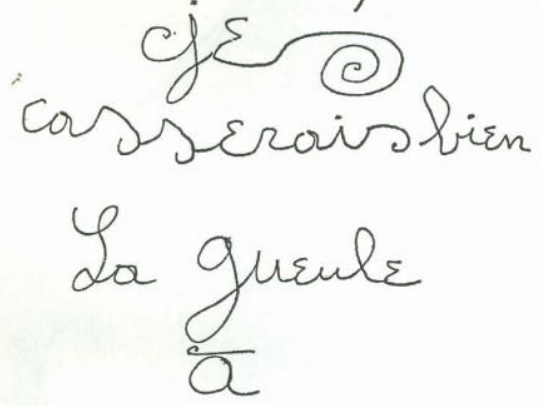

Tout lisgins
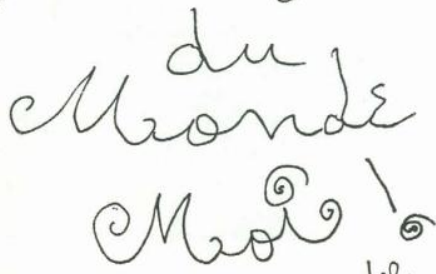

odilscroc 57 ,

44 


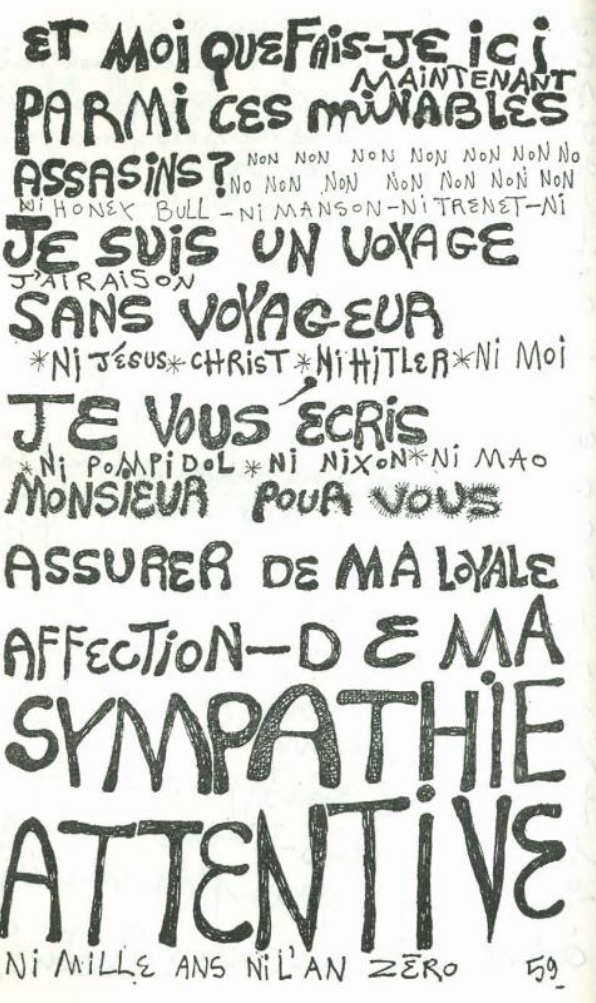

\section{Fragments d'un Sollers amoureux}

Érigée en mythe par Roberto Bolaño, portée aux nues par certaine critique trop lyrique ${ }^{6}$, Sophie Podolski a effectivement

\footnotetext{
${ }^{6}$ Le délire interprétatif de Collin, dans la notice qu'elle consacre à Podolski, pourrait de la sorte servir d'exemple-type à Stanley Fish quand celui-ci affirme que la littérature est avant tout créée par l'activité herméneutique : « S'il existe une œuvre qui résiste physiquement à l'attrait de la littérature, c'est celle de Sophie Podolski. Écriture instantanément publiée en première édition dans l'immédiateté de sa graphie, dans le prolongement de la main, qui décale et détourne les lignes, majuscule et minuscule les lettres, les lie, les
} 
de quoi retenir l'attention : au-delà du contenu virulent de son Pays où tout est permis, manifeste invectif contre le monde tel qu'il va et pour un trip universel, c'est la position institutionnelle de cette auteure sortie de nulle part et, à peine, de l'adolescence, qui intrigue. L'intégration rapide de la jeune poétesse à Tel Quel, l'un des derniers bastions de l'avant-garde, où elle est accueillie comme témoin et actrice d'un «bouleversement [qui] est en cours», est en réalité la conséquence d'un échange épistolaire. Dans une lettre envoyée de Bruxelles et datée du 29 novembre 1972, Podolski, lucide au sujet des lieux d'émergence et de rayonnement possibles d'une littérature underground, faisait parvenir à celui qui venait de publier Lois une manière de récit de rêve suivie d'un autoportrait kaléidoscopique ${ }^{7}$ :

phillip Solers,

Je vous jette des grands signes de loin en patin a roulettes. toute petite verte glissant sur le plafond en criant il flotte le monde etc... arrivée à côté de vous vous me racontez l'étrange aventure. nous décidons d'aller la trouver dans la crique ou elle se repose de la magnifiquence de ses derniers trajets. nous rions tous aspiré par la gluante fraîcheur du fumier orduriel.

hachure, se noue en dessins, s'épand en points, en grains, et dont l'imprimé ne peut rendre exactement compte. Mais il y a là une immédiateté toujours différée: celle de "l'autre main" pour reprendre l'expression de Maurice Blanchot. Rien d'aromatique en elle. Elle accompagne la vie, sautant d'un niveau à l'autre. Elle lie mais comme un gué. Elle déroule un ruban chaotique où les mots dansent, jetés en débandade, tantôt serrés, tantôt éclatés. Elle tient ensemble un certain espace. Elle occupe la page sous la garde incessante du non-dit de la mort. L'écriture de Sophie Podolski ne tend pas de miroirs. Si elle réfléchit et se réfléchit, c'est sans se figer dans la représentation. Elle va droit devant soi, dans un sens qui n'a pas de direction. Elle imprime ses traces sans se reprendre et sans se retourner : elle ne sera pas changée en statue de sel comme la femme de Lot, ni renvoyée aux enfers comme Eurydice. » (Collin, 1996, p. 475)

${ }^{7}$ Lettre publiée dans Luna Park, deuxième série, nº 5, mai 2009. 
(haletera un long spasme qui nous donne le sommeil). une nourrice explique notre oubli en carré noir. l'éttouffement soudain s'empare de la gorge et nous maintient siddéré sur un thème de magie noire où il $\mathrm{y}$ a victoire. Je ne crois pas que demain il faudra acheter ou ne pas acheter du pain ou autre chose. heu... c'est équivoque ceux qui parle du savoir savent connaître le savoir comme une methode énergique motrice. Je suis un merle parleur. je suis une flaque d'huile. Je suis un enfant assis par terre qui attend une recompense. tu as vu des cerveaux les uns dans les autres. c'est vrai qu'ils crient? l'escadron déboutonné des chats sur l'échelle se rendent chez moi. nous irons à la chorale des chameaux bouddhistes qui se rendent sur le bateau des naufragés de la névrose des blancs dégénérés. (nous ne sommes jamais que les assistants du vide). Je veux voir que nous sommes aucun mais au moins nous sommes deux chacun dans la ligne qui nous rejoint en nous internant deux dans chacun aussi Bien qu'autant deux dans aucun

\section{AMICALEMENT}

Sophie

Cette missive peu préoccupée des règles orthographiques fait écho au Pays où tout est permis. S'y retrouve, poussée à son paroxysme, une énonciation hésitante (avec le marqueur d'oralité « heu... » qui défie les codes des échanges épistolaires), nimbée d'un imaginaire hallucinatoire qui pourrait autant faire écho aux «cadavres exquis» surréalistes («nous rions tous aspiré par la gluante fraîcheur du fumier orduriel », « l'escadron déboutonné des chats sur l'échelle se rendent chez moi ») qu'à certaines constructions du Rimbaud des Illuminations ${ }^{8}$. Sont-ce

\footnotetext{
${ }^{8}$ Ainsi, la série de comparaisons à « un merle parleur », « une flaque d'huile », « un enfant assis par terre qui attend une recompense [sic]» n'est pas sans rappeler la litanie du quatrième volet du poème «Enfance », dans laquelle le «je» rimbaldien s'auto-identifie successivement au «saint, en prière sur la terrasse », au «savant au fauteuil sombre », au «piéton de la grand'route par les bois nains » et, surtout, affirme qu'il « serai[t] bien l'enfant abandonné sur
} 
ces petites traces de mimétisme qui ont attiré l'attention de Sollers, membre d'une " communauté interprétative » attentive aux signaux post-rimbaldiens ${ }^{9}$, sur cette jeune poétesse? Toujours est-il que, non content d'ouvrir à cette dernière les pages de Tel Quel et d'y escorter son texte, celui qui compte à l'époque parmi les principaux animateurs de la vie littéraire soutient en outre la réédition du Pays où tout est permis chez Belfond en lui accordant une préface. Sobrement intitulé «biologie » - titre délibérément ouvert à l'interprétation celle-ci se présente de la façon suivante :

L'expression prendre son pied est récente principe de versification tourné à l'envers je voudrais souligner que l'événement sur la page n'arrive jamais isolé reste à fond dans son ombre un arbre ne doit pas cacher la forêt les paroles dit artaud sont un limon qu'on n'éclaire pas du côté de l'être mais du côté de son agonie et encore on ne reproche pas à un écrivain un mot obscène parce qu'obscène on le lui reproche s'il est gratuit je veux dire plat et sans gris-gris et encore je ne supporterai pas éternellement que la sexualité universelle me carapace et me draine de la tête aux pieds voilà c'est tout à fait clair maintenant aucun vagin cuit recousu aucune fesse entrecul ne pourra barrer nos naissances donc elle dit la vie est castrante la mort décastrante formules condensées le rythme pense tout seul souffrance et encore souffrance ne prenez pas votre air amateur du menu folie pour les autres ça part de l'intenable ça y reviendra quelqu'un qui a mal au-delà du mal qui jouit par-delà le joui est facilement aujourd'hui l'objet d'un encadrement nouveau style profs journaux éditeurs maquereaux politiques fils de zofs sourds muets amputés en

la jetée partie à la haute mer, le petit valet, suivant l'allée dont le front touche le ciel ». La construction «nous ne sommes jamais que les assistants du vide », quant à elle, n'est pas sans faire écho au «nous ne serons jamais que des orphelins fiancés » du texte « Ouvriers ».

9 On doit pour rappel à Fish (2007) cette notion de «communauté interprétative », qui désigne un public conditionné à développer certains réflexes herméneutiques spécifiques en situation de lecture. 
tous genres c'est qu'on en a marre écrit-elle encore marre marre d'habiter sur quelque chose de pourri et non seulement archi-pourri mais en plus sphérique amusante cette prétention théorique de broder à côté d'la pratique la pensée pour eux ne ressemble pas au tonnerre finalement ils gardent leurs intonations d'autrefois on voit bien tout de suite ceux qui parmi eux vont renaître rendez-vous au prochain cycle bonsoir elle dit aussi je vois dans mon cerveau et pas ailleurs même si ailleurs il y a j'en ai marre marre des cons des culs des bites des couilles marre marre pensez-y des culs des bites des couilles marre marre pensez-y quand vous frez vos petites cochonneries du jour dans la nuit le tout écrit-elle encore est de savoir comment un poème inachevé peut-il achever un poème inachevé pour justement être un poète cette glace ne se prend pas dans la main le vice d'écrire au service du vice libre vice au service de la liberté vous ne croyez pas vraiment que la vérité va se dire en clair tout de même ceux qui le prétendent sont employés par la reproduction sans raison l'espèce peut en devenir élastique après tout le cercueil est à peine plus grand qu'un étron j'ai la manie dans mes rêves d'ouvrir des fenêtres voilà pourquoi j'aime ce livre qui n'est pas un livre quelques débiles croyant en majorité qu'il y a des livres ou encore que leur tête existe mais la faux passe est passée repasse chaque phrase aiguisée courbe tranchée la méthode consiste à ne jamais être d'un seul coup lucide oui exactement comme si on avait plusieurs vies d'un cynisme absolu un système d'autorégulation crache ton venin et autres conseils d'urgence parce que voyez-vous il est temps drôlement temps dans le temps de sauter à côté mais oui juste à côté de vous-mêmes je ne veux plus voir personne assis couché debout comme autrefois dans la loi nous n'avons jamais été des écrivains et pas non plus le contraire la musique dit beethoven est une révélation qui dépasse toute sagesse et toute philosophie de nouveau je vois la cour jaune. (1973b)

Encadrée par deux figures d'une certaine malédiction artistique (Artaud et Beethoven) censées autant faire rejaillir leur aura sur Sophie Podolski que placer celle-ci au même niveau que celles-là, la préface de Sollers s'inscrit directement, pour 
l'écrivain, dans une période de déstructuration poussée à son comble. Durant celle-ci, Sollers fait en effet paraître Lois (1972), mais également $H$ (1973), avant de mettre en route le chantier de Paradis, qui paraîtra en 1981. L'auteur se passionne alors pour une forme d'automatisme de la pensée, dont il veut rendre compte en s'affranchissant, notamment, des règles de syntaxe et de ponctuation : ce procédé a notamment pour effet d'effacer les contours des prises de paroles, à tel point que se distinguent mal, dans cette préface, les discours respectifs de Sollers et de Podolski - à tel point, pour le dire plus justement, que ce que dit celle-ci est directement épousé par celui-là. Dès lors, et même si certaines structures distributionnelles peuvent être plus ou moins isolées (« elle dit aussi je vois dans mon cerveau et pas ailleurs même si ailleurs il y a j'en ai marre marre des cons des culs des bites des couilles marre marre pensez-y des culs des bites des couilles marre marre pensez-y quand vous f'rez vos petites cochonneries du jour dans la nuit»), la logorrhée agressive de la poétesse se trouve appuyée par le préfacier, qui n'hésite pas lui-même à distribuer les insultes en s'en prenant aux faux aliénés et à ceux qui prétendent traiter et « encadrer » la folie (« ne prenez pas votre air amateur du menu folie pour les autres ça part de l'intenable ça y reviendra quelqu'un qui a mal au-delà du mal qui jouit par-delà le joui est facilement aujourd'hui l'objet d'un encadrement nouveau style profs journaux éditeurs maquereaux politiques fils de zofs sourds muets amputés en tous genres »). "Petite folle bienaimée », comme le dirait Baudelaire, Podolski importe aux yeux de Sollers parce que, dit-il, elle est authentique et révoltée jusqu'à l'os. S'il faut écouter cette jeune femme et lire ses textes, en somme, c'est parce qu'elle est incapable de supporter le monde putride qu'elle hante et qu'elle ose le dire. L'écrivain, du 
reste, trouve lui-même, en échange de bons procédés, un adjuvant à sa propre cause dans ce petit livre-monstre qu'il défend: si celui-ci s'en prend à tout le monde, ce n'est évidemment pas à lui, élu lucide capable d'échapper à la "planète de cons» que décrit Podolski, d'autant plus qu'il brandit lui-même cette dernière comme manifestation pure d'une forme de poésie organique concentrée sur l'essentiel.

Qu'un ouvrage porté par le discours d'escorte d'un auteur aussi influent que Sollers n'ait pas retenu outre mesure l'attention des historiographes de la littérature belge peut sembler étrange. Cette marque de reconnaissance transfrontalière constitue en effet un geste significatif au cœur d'une période post-1968 marquée, comme l'écrivent bien Benoît Denis et Jean-Marie Klinkenberg, par un « réaménagement des relations entre le centre [littéraire français] et la périphérie » $(2005$, p. 223). Les enjeux sont en réalité perceptibles dans différentes parties du champ littéraire: du côté dominant, représenté par l'Académie, la valorisation d'éléments francophones périphériques permet de témoigner du rayonnement du français au-delà de ses propres frontières ${ }^{10}$; du côté dominé, représenté par les dernières avant-gardes et qui nous concerne ici directement, l'ouverture à d'autres espaces permet de soutenir une croyance en l'«affaiblissement du concept même de littérature » (Denis et Klinkenberg, 2005, p. 224). Sophie Podolski, en cela, représente un intérêt institutionnel pour Sollers : terrifié aussi par l'idée de reproduire le ratage critique de ceux qui ont laissé filer des

\footnotetext{
10 Comme celle de Marguerite Yourcenar en 1980 (au troisième fauteuil), la nomination de Léopold Sédar Senghor à l'Académie française en 1983 (au seizième fauteuil), après une campagne de promotion menée pendant une dizaine d'années par Maurice Druon, en est un témoignage marquant.
} 
Rimbaud et Artaud, auteurs qu'il croit retrouver dans les lignes de sa protégée, le héraut de Tel Quel choisit de vanter le «mal écrire » délibéré de la jeune poétesse - qui se manifeste dans son mépris de l'orthographe (toutefois partiellement corrigée au moment de la mise en livre), de la syntaxe et de la ponctuation classiques, mais aussi dans son irrévérencieuse invective et dans l'omniprésence prosaïque de la drogue dans son texte - comme autant de traces d'un anti-académisme dont il est lui-même l'un des fers de lance et qu'il peut défendre avec d'autant plus de conviction qu'il se trouve des alliés imprévus et spontanés pour l'aider dans sa tâche.

\section{Bibliographie}

AnONYME. (2010), « Sophie Podolski : Le Pays où tout est permis ", sur Avant que j'oublie [blogue en ligne] 8 septembre, $<$ http://takecare-maready.blogspot.com/2010/09/sophiepodolski-le-pays-ou-tout-est.html>

Bolaño, Roberto. (2003), «Vagabonds en France et en Belgique », dans Des putains meurtrières, Paris, Christian Bourgois, coll. « Lettres », p. 99.

-. (2004), «Le Nil», dans Anvers, Paris, Christian Bourgois, coll. « Lettres », p. 29-30.

-. (2010), Les Détectives sauvages, Paris, Gallimard, coll. «Folio ». 
Collin, Françoise. (1996), "Sophie Podolski », dans Christiane Makward et Madeleine Cottenet-Hage (dir.), Le Dictionnaire littéraire des femmes de langue française, Paris, Karthala, p. $473-476$.

ConTEXTES. (2011), La Posture. Genèse, usages et limites d'un concept, no 8, janv., <http://contextes.revues.org/index4692.html>

DEnis, Benoît et Jean-Marie KLINKEnBERG. (2005), La littérature belge. Précis d'histoire sociale, Bruxelles, Labor, coll. « Espace nord».

Fish, Stanley. (2007), Quand lire c'est faire, Paris, Les Prairies ordinaires.

FRESÁN, Rodrigo. (2012), "Le détective sauvage et la planète des monstres », traduction d'Alexandre Civico, Le Believer, revue Inculte, $n^{\circ} 2$, été, p. 61-71.

GAUvin, Albert. (2009), Le Pays où tout est permis de Sophie Podolski, 20 sept., <http://www.pileface.com/sollers/article.php3?id article=918>

Lejeune, Philippe. (2005), Signes de vie. Le Pacte autobiographique 2, Paris, Seuil.

MeIzoz, Jérôme. (2007), Postures littéraires. Mises en scène modernes de l'auteur, Genève, Slatkine.

-. (2011), La Fabrique des singularités. Postures littéraires II, Genève, Slatkine.

Podolski, Sophie. (1973 [1972]), Le Pays où tout est permis, Paris, Belfond.

—. (2009), "Lettre à Philippe Sollers », Luna Park, deuxième série, $n^{\circ} 5$, mai. 
Simonet-TenAnt, Françoise. (2001), Le Journal intime, Paris, Nathan, coll. « $128 »$.

SOLLERS, Philippe. (1973a), "Le Pays où tout est permis », Tel Quel, nº 53, février.

—. (1973b), « Biologie », préface de Le Pays où tout est permis », Paris, Belfond, p. 11-13.

\title{
Résumé
}

Sorte de petit météore n'ayant pu mener une véritable trajectoire dans le monde des lettres, suicidée à vingt-et-un ans après la parution d'une œuvre sinueuse, Le Pays où tout est permis, Sophie Podolski se révèle l'une des incarnations contemporaines du mythe de la malédiction littéraire. Cet article se propose d'interroger la façon dont cette marginalité s'est construite sur les bases d'une virulente poétique de l'invective, qui rythme le recueil de la poétesse, mais s'est également renforcée dans les marges de ce dernier, par le discours d'escorte synchronique ou postérieur que lui ont consacré des auteurs comme Philippe Sollers et Roberto Bolaño.

\begin{abstract}
Sophie Podolski, who committed suicide at 21 years, after the publication of her only work Le Pays où tout est permis, turns one contemporary incarnations of the myth of "la malédiction littéraire". This paper aims to examine how this marginality is built on the foundations of a virulent poetic of invective, which dynamizes her book, but has also increased in the discourse about Le Pays où tout est permis from Philippe Sollers and Roberto Bolaño.
\end{abstract}

